\title{
MK-801 Exposure during Adolescence Elicits Enduring Disruption of Prefrontal E-I Balance and Its Control of Fear Extinction Behavior
}

\author{
Eden Flores-Barrera, ${ }^{*}$ Daniel R. Thomases, ${ }^{*}$ and Kuei Y. Tseng \\ Department of Anatomy and Cell Biology, College of Medicine, University of Illinois at Chicago, Chicago, Illinois 60614
}

Understanding how disruption of prefrontal cortex (PFC) maturation during adolescence is crucial to reveal which neural processes could contribute to the onset of psychiatric disorders that display frontal cortical deficits. Of particular interest is the gain of GABAergic function in the PFC during adolescence and its susceptibility to the impact of transient blockade of NMDA receptor function. Here we assessed whether exposure to MK-801 during adolescence in male rats triggers a state of excitatory-inhibitory imbalance in the PFC that limits its functional capacity to regulate behavior in adulthood. Recordings from PFC brain slices revealed that MK-801 exposure during adolescence preferentially reduces the presynaptic functionality of GABAergic activity over that of excitatory synapses. As a result, an imbalance of excitatory-inhibitory synaptic activity emerges in the PFC that correlates linearly with the GABAergic deficit. Notably, the data also suggest that the diminished prefrontal GABAergic function could arise from a deficit in the recruitment of fast-spiking interneurons by excitatory inputs during adolescence. At the behavioral level, MK-801 exposure during adolescence did not disrupt the acquisition of trace fear conditioning, but markedly increased the level of freezing response during extinction testing. Infusion of the $\mathrm{GABA}_{\mathrm{A}}$ receptor-positive allosteric modulator Indiplon into the PFC before extinction testing reduced the level of freezing response in MK-801-treated rats to control levels. Collectively, the results indicate NMDA receptor signaling during adolescence enables the gain of prefrontal GABAergic function, which is required for maintaining proper excitatory-inhibitory balance in the PFC and its control of behavioral responses.

Key words: adolescence; fast-spiking interneurons; fear conditioning; GABA; NMDA; prefrontal cortex

Significance Statement

A developmental disruption of prefrontal cortex maturation has been implicated in the pathophysiology of cognitive deficits in psychiatric disorders. Of particular interest is the susceptibility of the local GABAergic circuit to the impact of transient disruption of NMDA receptors. Here we found that NMDA receptor signaling is critical to enable the gain of prefrontal GABAergic transmission during adolescence for maintaining proper levels of excitatory-inhibitory balance in the PFC and its control of behavior.

\section{Introduction}

Adolescence often coincides with the age of onset for psychiatric disorders that display deficits in the prefrontal cortex (PFC) and associated cognitive functions (Kessler et al., 2007; Paus et al.,

\footnotetext{
Received Mar. 10, 2020; revised May 5, 2020; accepted May 11, 2020.

Author contributions: K.Y.T. designed research; E.F.-B., D.R.T., and K.Y.T. performed research; E.F.-B., D.R.T., and K.Y.T. analyzed data; K.Y.T. wrote the paper.

This research was supported by National Institute of Mental Health Grant R01-MH-086507 to K.Y.T. We thank Dr. J. Amiel Rosenkranz for assisting with setting up the behavior paradigm, and Dr. Adriana Caballero for thoughtful comments on the manuscript. This study was initiated at Chicago Medical School, Rosalind Franklin University of Medicine and Science.

${ }^{*}$ E.F.-B. and D.R.T. contributed equally to this work.

The authors declare no competing financial interests.

Correspondence should be addressed to Kuei Y. Tseng at tsengky@uic.edu.

https://doi.org/10.1523/JNEUROSCI.0581-20.2020

Copyright $\odot 2020$ the authors
}

2008; Gogtay et al., 2011). As the PFC continues to mature and its functional connectivity with afferent structures changes through adolescence (Caballero et al., 2016), the acquisition of cognitive abilities associated with adult behavior emerges (Casey et al., 2000; Best and Miller, 2010). Thus, understanding how PFC maturation occurs during adolescence will reveal key neural processes underlying normal development, and how their disruption could lead to the onset of mental disorders (Caballero et al., 2016; Caballero and Tseng, 2016).

Of particular interest is the maturation of PFC GABAergic function and its sensitivity to developmental disruption during adolescence (Caballero and Tseng, 2016). Recent evidence indicates that NMDA receptor transmission during adolescence is required for the acquisition of a GABAergic function in the PFC that regulates the impact of excitatory inputs (Thomases et al., 2013, 2014). In fact, the normal inhibitory control of ventral 
hippocampal and basolateral amygdalar inputs in the PFC is no longer present following adolescent exposure to the NMDA receptor antagonist MK-801 (Thomases et al., 2014). Of note, these two convergent PFC afferents are critical for the expression of cognitive function, including working memory (Tse et al., 2015), and the learning and extinction of conditioned fear responses (Sierra-Mercado et al., 2011; Gilmartin et al., 2012). Thus, transient disruption of NMDA receptor function during adolescence could trigger an enduring imbalance of inhibitory and excitatory activity in the PFC that reduces its functional capacity and behavioral control.

The goal of the present study is to reveal how adolescent exposure to MK-801 (Thomases et al., 2013, 2014) alters the balance of glutamatergic and GABAergic transmission in the PFC and its control of behavioral responses. To this end, we conducted whole-cell patch-clamp recordings to determine the extent of GABAergic and glutamatergic synaptic disruption occurring in the PFC following adolescent MK-801 exposure. At the behavioral level, we used a trace fear conditioning paradigm paired with pharmacological manipulations in the PFC to assess whether local excitatory-inhibitory (E-I) imbalance contributes to the behavioral deficit elicited by MK-801 during adolescence.

\section{Materials and Methods}

All experimental procedures were approved by the University of Illinois at Chicago and Rosalind Franklin University of Medicine and Science Institutional Animal Care and Use Committee in accordance with National Institutes of Health Guidelines for the Care and Use of Laboratory Animals. Upon arrival, male Sprague Dawley rats (Envigo) were allowed to habituate for at least $7 \mathrm{~d}$ before receiving any experimental manipulation. All rats were group housed (three per cage), and were kept under constant temperature $\left(21-23^{\circ} \mathrm{C}\right)$, humidity, and light/dark cycle with food and water available ad libitum. Both adolescent [postnatal day 35 (P35) to P40] and adult (P75-P80) rats were randomly assigned to receive daily noncontingent injections of either saline $(0.9 \%$ $\mathrm{NaCl})$ or $\mathrm{MK}-801(0.1 \mathrm{mg} / \mathrm{kg}$ in $0.9 \% \mathrm{NaCl}$, i.p.) for 5 consecutive days, and changes in electrophysiological and behavioral measures were determined from at least $25 \mathrm{~d}$ after the last injection, as in our previous studies (Thomases et al., 2013, 2014). All chemicals were purchased from Sigma-Aldrich with the exception of Indiplon, which was obtained from Tocris Bioscience.

Whole-cell patch-clamp recordings of IPSCs in the PFC. All brain slicing and patch-clamp recording procedures were conducted as previously described (Flores-Barrera et al., 2017). Briefly, layer V pyramidal neuron recordings were obtained from both the prelimbic and infralimbic PFC (350- $\mu \mathrm{m}$-thick coronal slices) at $33-35^{\circ} \mathrm{C}$ using a cesium-based internal solution containing $0.1 \%$ NEUROBIOTIN (Vector Laboratories) and the following (in mM): $140 \mathrm{CsCl}, 10$ HEPES, $2 \mathrm{MgCl}_{2}, 5 \mathrm{NaATP}, 0.6$ NaGTP, and 3 QX-314, at pH 7.23-7.28 and 280-282 mOsm. The recording aCSF contained $2 \mathrm{~mm}$ kynurenic acid or $10 \mu \mathrm{M} \mathrm{CNQX}+50 \mu \mathrm{M}$ $\mathrm{APV}$, to block fast glutamatergic transmission, and the following (in $\mathrm{mm}$ ): $122.5 \mathrm{NaCl}, 3.5 \mathrm{KCl}, 25 \mathrm{NaHCO}_{3}, 1 \mathrm{NaH}_{2} \mathrm{PO}_{4}, 2.5 \mathrm{CaCl}_{2}, 1 \mathrm{MgCl}_{2}$, 20 glucose, and 1 ascorbic acid, at pH 7.40-7.43 and 295-305 mOsm. Both spontaneous and evoked $\mathrm{GABA}_{\mathrm{A}}$ receptor-mediated IPSC were recorded in voltage-clamp mode at a holding potential of $-70 \mathrm{mV}$. In some cases, the $\mathrm{Na}^{+}$channel blocker TTX $(0.5 \mu \mathrm{M})$ was included in the recording solution to obtain miniature IPSC. Only neurons exhibiting stable baseline activity ( $>15 \mathrm{~min}$ duration) were included. The mean IPSC frequency was estimated from at least two noncontiguous epochs of $60 \mathrm{~s}$ recordings. Changes in locally evoked IPSC were assessed by means of electrical stimulation (every $10 \mathrm{~s}$ ) using a Teflon-coated bipolar electrode placed $\sim 200 \mu \mathrm{m}$ from the cell body along the apical dendritic axis. The stimulation intensity $(300 \mu$ s square pulses in the $10-50 \mu \mathrm{A}$ range) was titrated from the minimum current required to elicit an IPSC response with a failure rate of $\sim 50 \%$ using a paired-pulse protocol (50 ms interval, every $10 \mathrm{~s}$ ).
Recordings of excitatory and inhibitory synaptic events within a single pyramidal neuron. All recordings were conducted from layer $\mathrm{V}$ pyramidal neurons of the prelimbic PFC using a low-chloride-based internal solution and an external solution free of glutamate and GABA blockers to enable concurrent acquisition of excitatory and inhibitory synaptic currents at the single-cell level (Flores-Barrera et al., 2017). The internal solution also contained 0.1\% NEUROBIOTIN (Vector Laboratories) and the following (in mM): $10 \mathrm{CsCl}, 130$ gluconic acid, $10 \mathrm{HEPES}, 2 \mathrm{MgCl}_{2}, 5$ NaATP, 0.6 NaGTP, and 3 QX-314, at pH 7.23-7.28 and 280-282 mOsm). Both spontaneous glutamatergic and GABAergic synaptic events can be readily assessed by recording the frequency of postsynaptic currents (PSC) at the $-60 \mathrm{mV}$ ( PSC $_{-60 \mathrm{mV}}$ ) and $+15 \mathrm{mV}\left(\mathrm{PSC}_{+15 \mathrm{mV}}\right.$ ) holding potentials, respectively. As described above, only neurons with at least $15 \mathrm{~min}$ of stable baseline activity were included for analyses. The frequency of $\mathrm{PSC}_{-60 \mathrm{mV}}$ and $\mathrm{PSC}_{+15 \mathrm{mV}}$ events from at least two noncontiguous epochs of $60 \mathrm{~s}$ each was compared with equivalent measures taken $10 \mathrm{~min}$ after bath application of the $\mathrm{GABA}_{\mathrm{A}}$ receptor antagonist picrotoxin $(20 \mu \mathrm{M})$.

Whole-cell patch-clamp recordings of fast-spiking interneurons in the $P F C$. All recordings were conducted from layer $\mathrm{V}$ fast-spiking interneurons (FSI) of the prelimbic PFC using a potassium-based internal solution, as previously described (Caballero et al., 2014a). Briefly, 5-10 min after obtaining the whole-cell configuration in current-clamp mode, a set of somatic current pulses was applied to measure changes in neuronal excitability and spike-frequency adaptation of the recorded cell. Recordings were then switched to voltage-clamp mode (holding potential, $-70 \mathrm{mV}$ ), and the number of spontaneous EPSC was collected. Only cells that remained stable for at least $20 \mathrm{~min}$ after obtaining the whole-cell configuration were included. As in our previous study (Caballero et al., 2014a), the mean frequency of spontaneous EPSC was estimated from at least two noncontiguous 60 -s-duration samples.

Trace fear conditioning and extinction. We used a fear-conditioning protocol modified from Zhang and Rosenkranz (2013). Briefly, all testing chambers were housed in sound-attenuating cabinets (Ugo Basile) with a constant white noise-generating fan (60-70 dB; Scientific Design). The trace fear-conditioning phase consisted of $120 \mathrm{~s}$ of habituation followed by 5 pairings of a neutral tone $(10 \mathrm{~s}, 1500 \mathrm{~Hz}, 85 \mathrm{~dB})$ paired with a footshock ( $1 \mathrm{~s}, 0.3 \mathrm{~mA})$ at a delay of $20 \mathrm{~s}$ from the end of the tone. Conditioning trials were presented using a pseudorandom intertrial interval of 240-280 s. After conditioning, rats were returned to their home cage for $24 \mathrm{~h}$. Extinction trials began the next day in a visually and tactilely distinct chamber. Following a 120 $\mathrm{s}$ habituation period, the conditioned tone $(20 \mathrm{~s})$ was presented 14 times $(60 \mathrm{~s} /$ trial $)$ without footshock to enable the extinction of fear memory. The acquisition of fear extinction is typically revealed by the degree of conditioned freezing (i.e., lack of nonrespiratory movement $>0.5 \mathrm{~s}$ ) to the tone that diminishes over repeated trials. Quantification of time spent freezing (percentage of freezing) was determined from trial to trial using an infrared camera connected to the ANY-maze analysis software (Stoelting).

PFC infusion of Indiplon. Ten days before trace fear-conditioning experiments, rats were implanted with bilateral infusion cannulas targeting the medial PFC region. Rats were first placed in a chamber that is saturated with $5 \%$ isoflurane mixed with oxygen (Somnosuite Unit, Kent Scientific). The level of anesthesia was monitored by pinching the hindfoot using forceps and assessing the absence of the withdrawal reflex (hindlimb compression reflex). Before mounting the rats in the stereotaxic apparatus, the head was shaved and scrubbed with povidone iodine (7.5\%; Medline) and 75\% alcohol. The skin overlying the skull was then infiltrated with $2 \%$ lidocaine hydrochloride (containing 1:100,000 epinephrine) to diminish possible discomfort following the surgical procedure. Rats were then placed in a stereotaxic frame using nonrupture ear bars (Kopf), and the skull was exposed. Isoflurane anesthesia (2-3\%) was maintained using a Somnosuite Unit with the body temperature kept within the $37-38^{\circ} \mathrm{C}$ range (TCAT-2LV controller Physitemp). Two skull screws (catalog \#0-80-X3-32, Plastics One) were inserted into the skull to anchor the acrylic cement head assembly, and two burr holes were then drilled in the skull above the medial PFC region to enable placement 

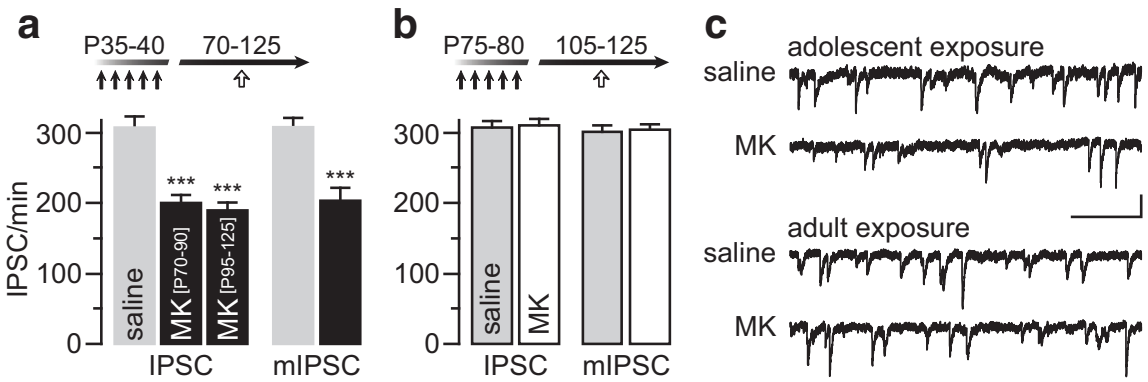

Figure 1. Adolescent MK-801 exposure reduces the frequency of IPSC onto prefrontal pyramidal neurons. $\boldsymbol{a}$, MK-801 exposure during adolescence (black arrows, P35-P40) diminished the frequency of IPSC recorded from layer V pyramidal neurons in the PFC. Relative to saline-treated controls $(n=19)$, the IPSC frequency in MK-801-treated rats remained reduced through adulthood from P70-P90 $(n=14)$ to P95-P125 ( $n=9$; ${ }^{* * *} p<0.001$ vs saline, unpaired $t$ test). A similar reduction in miniature IPSC (mIPSC) was found between saline-treated $(n=8)$ and MK-801-treated $(n=12)$ groups $\left({ }^{* * *} p<0.001\right.$ vs saline, unpaired $t$ test). $\boldsymbol{b}$, In contrast, the frequency of IPSC recorded from rats that received MK-801 during adulthood (black arrows, P75-P80; $n=11)$ resembled that of saline-treated controls $(n=7)$. Similarly, the frequency of mIPSC remained unchanged following adult MK-801 exposure ( $n=7-8$ /group). c, Example traces of IPSC recorded from layer $V$ pyramidal neuron illustrating the age-dependent impact of MK-801. Calibration: 20 pA, 500 ms.
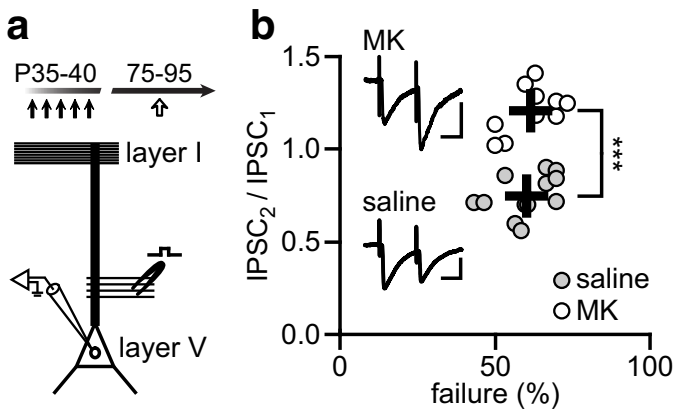

Figure 2. Paired-pulse facilitation of IPSC following adolescent MK-801 exposure. $\boldsymbol{a}$, Time line and diagram illustrating the experimental design used to elicit IPSC in layer V pyramidal neurons with a paired-pulse protocol of minimal stimulation. $\boldsymbol{b}$, Pyramidal neurons in the PFC of saline-treated control rats $(n=12)$ exhibited a paired-pulse suppression (IPSC $2 /$ IPSC $_{1}$ $<1.0$ ) response at a $50 \mathrm{~ms}$ interstimulation interval. In contrast, all neurons recorded from adult rats that received MK801 treatment during adolescence showed a paired-pulse facilitation (IPSC $2 /$ IPSC $_{1}>1.0$ ) response ( $n=10 ;{ }^{* * *} p<0.001$ vs saline, unpaired $t$ test). Insets are example traces of evoked IPSC illustrating the impact of adolescent MK-801 exposure. Calibration: $10 \mathrm{pA}, 25 \mathrm{~ms}$.

of a stainless steel $26 \mathrm{G}$ guide cannula (Plastics One) bilaterally to the prelimbic area at a $25^{\circ}$ angle ( $3.0 \mathrm{~mm}$ anterior to bregma; $2.8 \mathrm{~mm}$ lateral; 3.5 $\mathrm{mm}$ below from the brain surface). After securing the guide cannulas with acrylic cement (Stoelting), a 33G dummy cannula was screwed into each of the guide cannula to prevent clogging. One day before fear conditioning testing, the dummy cannulas were replaced with those protruding $0.5 \mathrm{~mm}$ beyond the tip of the guide cannulas. All PFC infusions $(0.8 \mu \mathrm{l}$ at $0.5 \mu \mathrm{l} /$ $\mathrm{min}$ ) of aCSF-containing vehicle or the $\mathrm{GABA}_{\mathrm{A}}$ positive allosteric modulator Indiplon ( $10 \mu \mathrm{M}$ in $0.04 \%$ DMSO) were delivered $20-25 \mathrm{~min}$ before the start of fear extinction trials. This dose of Indiplon was chosen because it can restore the balance of excitatory-inhibitory transmission resulting from a presynaptic GABAergic disruption (Flores-Barrera et al., 2017) and prefrontal GABAergic function in MK-801-treated rats in vivo (Thomases et al., 2013, 2014). Cannula placement was determined in Nissl-stained PFC sections, as previously described (Thomases et al., 2013).

Statistical analysis. Data were summarized as the mean \pm SEM, and differences among experimental conditions (within- and between-subject design) were considered statistically significant when the $p$ value was $<0.05$ (StatSoft). Paired $t$ test was preferred for two-group comparison following a within-subject design involving a single continuous variable (e.g., predrug vs postdrug application). Two-way ANOVA was used for testing between-subject comparisons along three or more dependent variables.

\section{Results}

We first determined whether MK-801 exposure during adolescence (P35-P40) elicits enduring changes in GABAergic transmission in the PFC by means of ex vivo electrophysiological recordings of IPSC onto layer V pyramidal neurons. Data obtained from prelimbic (saline: $304.8 \pm 9.9 / \mathrm{min}, n=14$; MK-801: $201.8 \pm$ 9.9/min, $n=19$ ) and infralimbic (saline: 303.1 \pm 16.5/min, $n=5$; MK-801: $205.1 \pm$ $16.3 / \mathrm{min}, n=4)$ regions were pooled because no apparent differential effect of MK-801 was observed. Overall, the frequency of IPSC (both spontaneous and miniature) was markedly diminished following adolescent MK-801 exposure (Fig. 1a), an effect that was not present when MK-801 treatment occurred in adulthood (P75-80; Fig. 1b). The reduced IPSC frequency observed in adolescent MK-801-treated rats is long lasting as it was detectable through adulthood from P70 to P125 (Fig. $1 a-c$ ). Such age-dependent disruption by MK801 was not associated with changes in IPSC amplitude (saline: $22.2 \pm 1.2 \mathrm{pA}$; MK-801: $21.7 \pm 0.9 \mathrm{pA}$ ), rise time (saline: $1.9 \pm 0.1 \mathrm{~ms} ;$ MK-801: $1.9 \pm 0.1 \mathrm{~ms}$ ), decay (saline: $15.6 \pm$ $0.7 \mathrm{~ms}$; MK-801: $15.3 \pm 1.2 \mathrm{~ms}$ ), and half-width duration (saline: $6.4 \pm 0.3 \mathrm{~ms}$; MK-801: $6.3 \pm 0.6 \mathrm{~ms}$ ). Furthermore, data obtained from locally evoked IPSC using a protocol of pairedpulse stimulation further revealed a presynaptic disruption of GABAergic transmission (Fig. 2). While pyramidal neurons recorded from saline-treated controls exhibited a typical paired-pulse suppression (IPSC ${ }_{2} / \mathrm{IPSC}_{1},<1.0$ ), a pairedpulse facilitation (IPSC $\mathrm{IPSC}_{1},>1.0$ ) emerged in the adolescent MK-801-treated group (Fig. 2b). Collectively, these results point to a presynaptic mechanism underlying the prefrontal GABAergic deficit observed following adolescent MK-801 exposure.

Adolescent MK-801 exposure could also elicit an enduring glutamatergic disruption in the PFC. Thus, the E-I balance of synaptic activity within a single pyramidal neuron would remain unaltered if a similar reduction in glutamatergic drive occurs following adolescent MK-801 treatment. To address this, layer V pyramidal cells from the prelimbic region were recorded in adulthood (P75-P95) using a protocol that enables concurrent acquisition of GABAergic and glutamatergic synaptic activity (Fig. 3; Flores-Barrera et al., 2017). Data reveal that adolescent MK-801 exposure diminished the frequency of $\mathrm{GABA}_{\mathrm{A}}$-sensitive postsynaptic currents $\left(\mathrm{PSC}_{+15 \mathrm{mV}}\right.$ ) without altering the number of glutamatergic (PSC $-60 \mathrm{mV}$ ) events (Fig. $3 a, b$ ). As a result, all pyramidal neurons recorded from the adolescent MK-801treated group exhibited a significantly higher E/I ratio (Fig. $3 c$ ) in a manner that correlates linearly with the frequency of $\mathrm{GABA}_{\mathrm{A}}$-sensitive $\mathrm{PSC}_{+15 \mathrm{mV}}$ (Fig. $3 d$ ). These results indicate MK-801 exposure during adolescence preferentially reduces PFC GABAergic transmission over that of glutamatergic synapses.

The long-lasting E-I imbalance observed in the PFC of adolescent MK-801-treated rats could arise from a deficient recruitment of local GABAergic function by excitatory inputs during adolescence (Cass et al., 2013; Thomases et al. 2013, 2014; Caballero et al., 2014b). Of particular interest is the facilitation of glutamatergic transmission onto parvalbumin -positive FSI (Caballero et al., 2014a), the most abundant class of GABAergic 

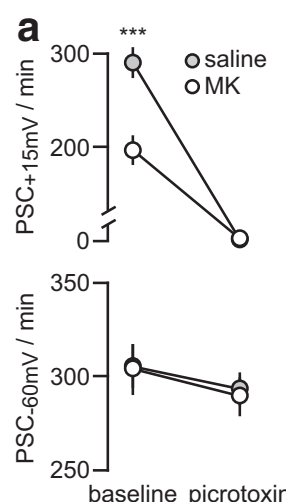

(20 uM)
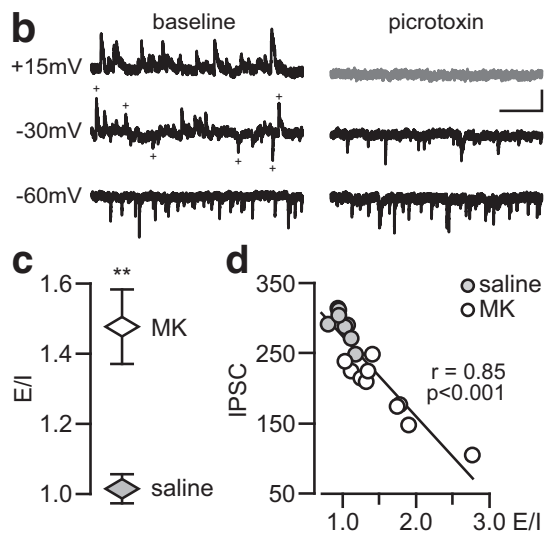

Figure 3. Prefrontal E-I balance is lost following adolescent MK-801 exposure. $\boldsymbol{a}$, Recordings of a spontaneous PSC from inhibitory (top, PSC $+15 \mathrm{mv}$ ) and excitatory (bottom, PSC $60 \mathrm{mv}$ ) synapses onto layer V pyramidal neurons in the PFC of adult rats (i.e., P75-P95). Relative to saline-treated controls $(n=8)$, the frequency of $\mathrm{PSC}_{+15 \mathrm{mv}}$ was significantly diminished following adolescent (P35-P40) MK-801 exposure $\left(n=10 ;{ }^{* *} p<0.001\right.$, unpaired $t$ test). Such a difference was not observed when cells were held at $-60 \mathrm{mV}$. Of note, bath application of the $\mathrm{GABA}_{\mathrm{A}}$ receptor antagonist picrotoxin $(20 \mu \mathrm{m}, 10 \mathrm{~min})$ completely abolished the $\mathrm{PSC}_{+15 \mathrm{mV}}$ events, while synaptic activity at the $-60 \mathrm{mV}$ holding potential remained largely unaltered. $\boldsymbol{b}$, Example traces of a spontaneous PSC recorded from a layer V pyramidal cell at three different holding potentials before and after bath application of picrotoxin. Calibration:

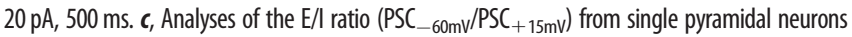
revealed an imbalance toward excitation in the adult PFC of adolescent MK-801-treated rats $\left({ }^{* *} p<0.01\right.$ vs saline, unpaired $t$ test). $\boldsymbol{d}$, Regression analyses revealing a significant correlation between the frequency of $\mathrm{PSC}_{+} 15 \mathrm{mv}$ (i.e., IPSC) and the $\mathrm{E} / \mathrm{I}$ ratio within single pyramidal cells.

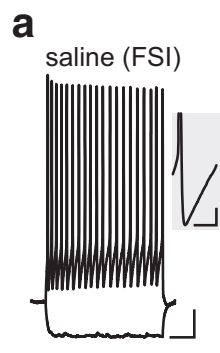

C saline (FSI)

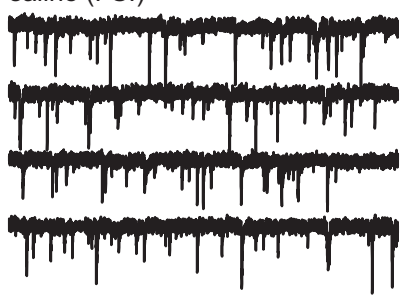

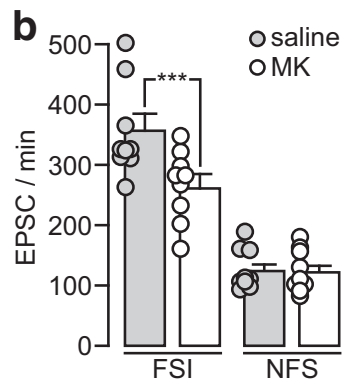

MK (FSI)

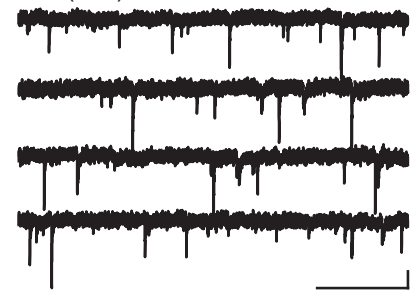

Figure 4. Adolescent MK-801 exposure reduces the frequency of EPSC onto prefrontal FSI. $\boldsymbol{a}$, Example traces of FSI recorded from saline-treated and MK-801-treated rat PFCs illustrating the typical nonadapting firing response to somatic current depolarization (160 pA, $500 \mathrm{~ms}$; calibration: $10 \mathrm{mV}, 100 \mathrm{~ms}$ ) and the prominent afterhyperpolarization potential (inset; calibration: $6 \mathrm{mV}$, $10 \mathrm{~ms})$. $\boldsymbol{b}$, Bar graph summarizing the number of spontaneous EPSC recorded from FSI and nonfast-spiking interneurons (NFS) in the PFC of adult rats (P75-P115) that underwent saline or MK801 treatment during adolescence (P35-P40). Relative to the saline-treated control group, a marked reduction in EPSC frequency was observed only in FSI recorded from adolescent MK-801-treated rats ( ${ }^{* *} p<0.0001$, unpaired $t$ test). c, Example traces of spontaneous EPSC recorded from FSI, illustrating the impact of the adolescent MK-801 exposure shown in $\boldsymbol{b}$. Calibration: 10 pA, 500 ms.

cells in the rat prelimbic PFC (Gabbott et al., 1997). Thus, we conducted whole-cell patch-clamp recordings and determined whether MK-801 exposure during adolescence disrupts the level of EPSC in FSI. Typically, FSI can be easily distinguished by the
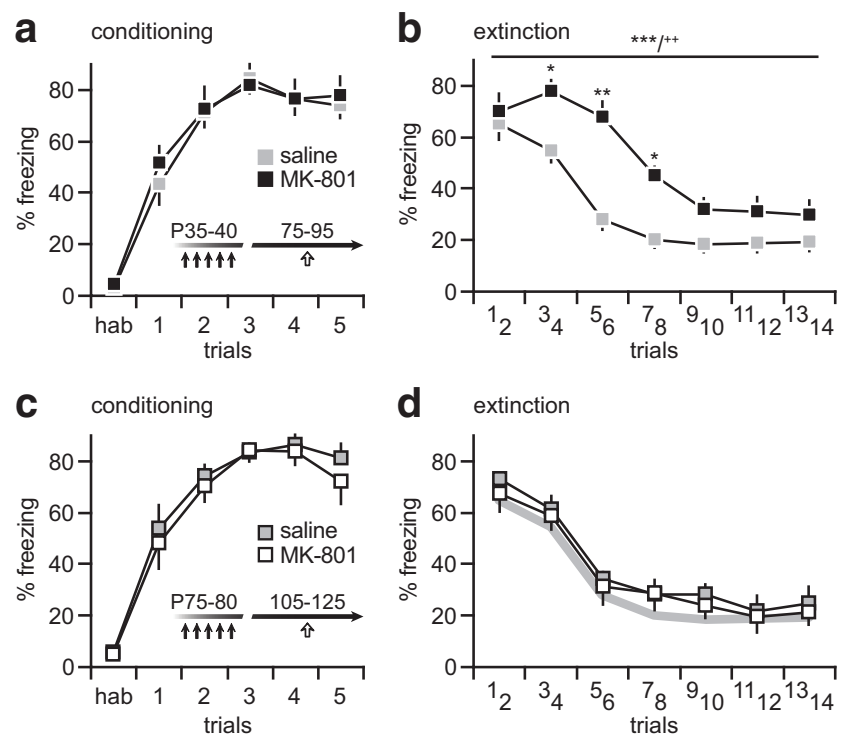

Figure 5. Adolescent MK-801 exposure increases the level of freezing response during extinction. $\boldsymbol{a}$, Relative to saline-treated controls $(n=7)$, MK-801 exposure during adolescence (black arrows, P35-P40; $n=8$ ) did not disrupt the acquisition of trace fear conditioning in adulthood (white arrow, P75-P95), as revealed by the percentage of freezing. $\boldsymbol{b}$, However, the pattern of freezing response during extinction ( $24 \mathrm{~h}$ after conditioning) is markedly altered in adolescent MK-801-treated rats. Typically, the level of freezing response to the conditioned tone diminishes over repeated trials (14 trials of $60 \mathrm{~s}$ each, see Materials and Methods for details), as observed in saline-treated controls rats. This was not the case in rats that underwent adolescent MK-801 exposure, which exhibited a higher degree of freezing behavior during extinction (main effect of treatment: $F_{(1,91)}=52.1$, ${ }^{* * *} p<0.0001$; main effect of trials: $F_{(6,91)}=35.7,{ }^{* *} p<0.0001$; treatment $\times$ trials interaction: $F_{(6,91)}=3.1$, $++p<0.01$; tw0-way ANOVA), in particular from trials $3-8\left({ }^{*} p<0.05,{ }^{* *} p<0.005\right.$, Tukey's post hoc test). c, Similar to the adolescent cohort shown in $\boldsymbol{a}$, rats that received saline $(n=6)$ or MK-801 $(n=7)$ injections during adulthood (i.e., P75-P80) exhibited a normal pattern of freezing response during the acquisition of fear conditioning (white arrow, P105-P125). d, Interestingly, MK-801 exposure during adulthood failed to disrupt the normal pattern of trace fear extinction. The gray line depicts the extinction profile of salinetreated controls shown in $\boldsymbol{b}$.

characteristic nonadapting firing response to somatic depolarization and a prominent afterhyperpolarization potential (saline: 23.6 $\pm 1.0 \mathrm{mV}$; MK-801: 22.3 $\pm 0.9 \mathrm{mV}$; Fig. 4a; Caballero et al., 2014a). Relative to saline-treated controls, FSI recorded from adolescent MK-801-treated rats exhibited a lower frequency of EPSC (Fig. 4b,c) without any detectable changes in EPSC amplitude (saline: $15.4 \pm 0.7 \mathrm{pA}$; MK-801: $16.6 \pm 1.7 \mathrm{pA}$ ), resting membrane potential (saline: $-66.2 \pm 1.4 \mathrm{mV}$; MK-801: $-66.3 \pm$ $2.1 \mathrm{mV}$ ), or input resistance (saline: $204.2 \pm 18.7 \mathrm{M} \Omega$; MK-801: $206.7 \pm 18.7 \mathrm{M} \Omega$ ). Such a deficit in EPSC frequency resembles that of naive juvenile animals (Caballero et al., 2014a), suggesting that NMDA receptor function during adolescence is needed to enable normal levels of excitatory transmission onto FSI in adulthood. Of note, adolescent MK-801 exposure did not alter the frequency of EPSC in non-FSI (Fig. 4b).

The GABAergic deficit observed in the PFC following adolescent MK-801 exposure is associated with a disruption of prefrontal processing of afferent transmission, in particular those originated from the ventral hippocampus and basolateral amygdala (Thomases et al., 2014). Of note, both intact PFC and its major afferents are required for optimal performance in trace fear memory tasks (Gilmartin et al., 2012, 2014). Thus, we used a trace fear-conditioning paradigm to assess the extent by which MK-801 exposure during adolescence impacts behavior in adulthood. Data indicate that adolescent MK-801 exposure did not 

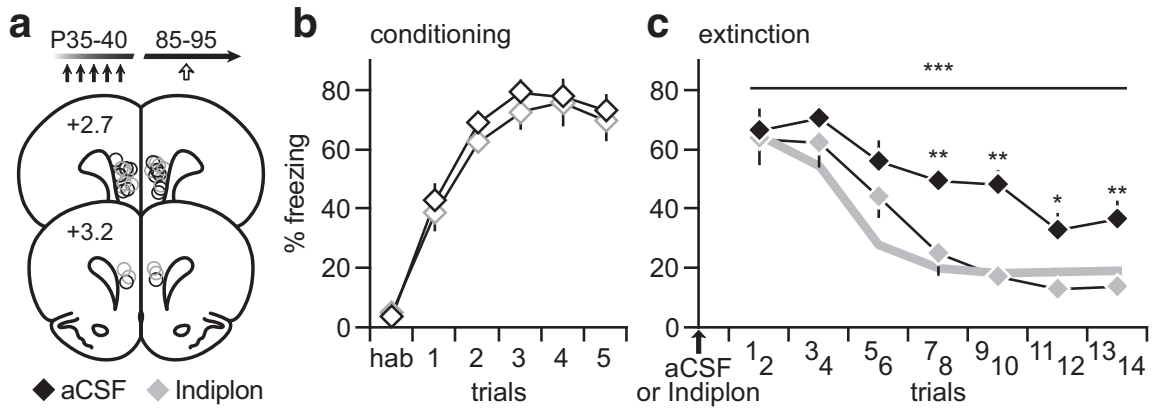

Figure 6. Prefrontal infusions of Indiplon normalized the behavioral deficit elicited by adolescent MK-801 treatment. $\boldsymbol{a}$, Diagram of coronal brain sections illustrating all PFC infusion sites of aCSF and Indiplon (10 $\mu \mathrm{M})$. All PFC infusions were performed 20 25 min prior to behavioral testing. $\boldsymbol{b}$. Data summarizing the acquisition of trace fear conditioning from adolescent MK-801-treated rats assigned to receive either aCSF $(n=9)$ or Indiplon $(n=8)$ on the next day. c, Relative to aCSF controls, PFC infusion of Indiplon significantly improved the fear extinction performance and reduced the percentage of freezing (main effect of PFC infusion: $F_{(1,105)}=35.6,{ }^{* *} p<0.0001$; two-way ANOVA), in particular from trials $7-14\left({ }^{*} p<0.05,{ }^{* *} p<0.005\right.$, Tukey's post hoc test). The extinction profile of saline-treated controls (gray line) shown in Figure $5 b$ was included for comparison.

disrupt the acquisition of trace fear conditioning in adulthood (Fig. 5a). However, MK-801-treated rats exhibited an increased freezing response to the conditioned tone during extinction testing (Fig. 5b). This was not the case in rats that underwent MK801 exposure during adulthood, which showed a comparable degree of freezing behavior as saline-treated controls in both acquisition and extinction (Fig. $5 c, d$ ). These results indicate that the resulting prefrontal GABAergic deficit may contribute to the agedependent behavioral disruption elicited by adolescent MK-801 exposure. To test this hypothesis, PFC infusion of the $\mathrm{GABA}_{\mathrm{A}}$ positive allosteric modulator Indiplon $(10 \mu \mathrm{M})$ was delivered $20-$ 30 min prior to extinction testing (Fig. $6 a$ ) to enhance prefrontal GABAergic transmission (Flores-Barrera et al., 2017) in adolescent MK-801-treated rats. Relative to aCSF controls, PFC infusion of Indiplon significantly reduced the freezing response in MK-801treated rats to control levels (Fig. 6b). Collectively, the results provide a mechanistic link between deficits in prefrontal GABAergic function and the age-dependent behavioral disruption observed following adolescent MK-801 exposure.

\section{Discussion}

In the present study, we found that the enduring and age-dependent impact of MK-801 exposure during adolescence results from a preferential disruption of GABAergic over glutamatergic synapses in the PFC. Consequently, the prefrontal E-I balance is lost in a manner that correlates linearly with the extent of the local GABAergic deficit. At the behavioral level, the enhanced freezing response observed in MK-801-treated rats during extinction was reduced to control levels following PFC infusion of the $\mathrm{GABA}_{\mathrm{A}}$ positive allosteric modulator Indiplon. Together, the data reveal that NMDA receptor signaling during adolescence is needed to enable the developmental gain of prefrontal GABAergic function, which in turn is required for maintaining proper E-I balance in the PFC and support behavioral responses in adulthood.

While both prefrontal GABAergic and glutamatergic processes undergo functional remodeling during adolescence (Caballero et al., 2016), it is the local GABAergic circuit that becomes labile to MK-801 exposure during this developmental period. In fact, only the gain of GABAergic function was interrupted by MK-801, a disruption that was not apparent when the treatment occurred in adulthood. Data obtained from adolescent MK-801-treated rats also point to a presynaptic mechanism underlying the PFC GABAergic deficit, a level of GABAergic transmission resembling that of naive juveniles (Cass et al., 2014). This is consistent with our in vivo studies showing a limited recruitment of GABAergic function by PFC inputs in adolescent MK-801-treated rats, while the excitatory component of afferentdriven responses remains intact (Thomases et al., 2013, 2014; FloresBarrera et al., 2014). Thus, any disruption of NMDA receptor signaling during adolescence (e.g., environmental or genetic factors; Do et al., 2009; Kantrowitz and Javitt, 2010) is expected to preferentially impact the maturation of prefrontal GABAergic circuits and compromise the E-I balance of PFC output in adulthood.

Consistent with previous findings, a functional impairment of GABAergic interneurons containing the calcium-binding protein parvalbumin (or FSI) can lead to PFC disinhibition, as seen in several rodent models of psychiatric disorders (Tseng et al., 2009; O’Donnell, 2011; Nakazawa et al., 2012). Typically, the susceptibility and functionality of parvalbumin-positive FSI are thought to be associated with the levels of oxidative stress (Powell et al., 2012; Hardingham and Do, 2016; Steullet et al., 2016) and parvalbumin expression (Caballero and Tseng, 2016). In the PFC, changes in parvalbumin during adolescence can define the functionality of FSIs and impact prefrontal output in adulthood (Caballero et al., 2020). Parallel to the acquisition of adult levels of parvalbumin in the PFC is the facilitation of glutamatergic transmission onto FSI (Caballero et al., 2014a), a developmental event that failed to occur following adolescent MK-801 exposure. It is possible that NMDA receptor transmission in the PFC from multiple long-range inputs (Bogart and O'Donnell, 2018) is needed to strengthen the enhanced glutamatergic drive onto FSI during adolescence (Caballero et al., 2016; Caballero and Tseng, 2016). Although NMDA transmission is lower than that of AMPA receptors in FSI (Wang and Gao, 2010; Rotaru et al., 2011; Bogart and O’Donnell, 2018), it does subserve a large phenotypic change that allows these GABAergic interneurons to transition into an "adult" profile. One such NMDA receptor-dependent event is the expression of parvalbumin itself (Kinney et al., 2006; Behrens et al., 2007), which begins to reach adult levels during adolescence to strengthen FSI activity and the gain of GABAergic transmission in the PFC (Caballero et al., 2020). In this regard, the absence of afferent-mediated NMDA transmission during adolescence will arrest PFC GABAergic development, specifically that of FSI, at a stage that provides insufficient inhibition to pyramidal neurons. Thus, we propose that the E-I imbalance observed in the PFC of adolescent MK-801-treated rats emerges from a failure to recruit excitatory inputs to FSI normally enabled by NMDA receptor signaling.

Prefrontal processing of afferent information and its impact on behavior is likely to be disrupted if the maturation of GABAergic circuits in the PFC is interrupted during adolescence. Of particular interest is the frequency-dependent inhibition of hippocampal and amygdalar inputs by the PFC, which is markedly reduced following adolescent MK-801 exposure (Thomases et al., 2013, 2014). Accordingly, PFC regulation of cognitive behaviors requiring integration of these inputs, such as the 
expression and extinction of conditioned fear responses (Phillips and LeDoux, 1992; Ishikawa and Nakamura, 2003; AngladaFigueroa and Quirk, 2005; Sierra-Mercado et al., 2006, 2011; Milad et al., 2007; Gilmartin et al., 2012; Sotres-Bayon et al., 2012), are expected to be compromised. This was indeed the case when rats were treated with MK-801 during adolescence and tested in adulthood, as revealed by an enhanced freezing response to the conditioned tone during extinction. Data obtained from PFC infusions of Indiplon further indicate that the abnormal extinction behavior observed in MK-801-treated rats could arise from a prefrontal GABAergic deficit. Remarkably, the strengthening of $\mathrm{GABA}_{\mathrm{A}}$ function restored the ability of ventral hippocampal inputs to drive PFC inhibition in MK-801treated rats in vivo (Thomases et al., 2013, 2014). Altogether, it is conceivable that the discrete behavioral disruption observed during extinction results from a deficient recruitment of PFC GABAergic activity by ventral hippocampal inputs during adolescence.

Collectively, the results presented here indicate that NMDA receptor signaling during adolescence is required for the development of a critical level of prefrontal GABAergic activity into adulthood. Failure to achieve adult levels of GABAergic function will result in a long-lasting imbalance of excitatory and inhibitory transmission in the PFC. Consequently, prefrontal regulation of behavioral responses requiring integration of ventral hippocampal and basolateral amygdalar inputs will be limited (Thomases et al., 2014), as seen in psychiatric disorders affecting cognitive and affective domains.

\section{References}

Anglada-Figueroa D, Quirk GJ (2005) Lesions of the basal amygdala block expression of conditioned fear but not extinction. J Neurosci 25:96809685.

Behrens MM, Ali SS, Dao DN, Lucero J, Shekhtman G, Quick KL, Dugan LL (2007) Ketamine-induced loss of phenotype of fast-spiking interneurons is mediated by NADPH-oxidase. Science $318: 1645-1647$.

Best JR, Miller PH (2010) A developmental perspective on executive function. Child Dev 81:1641-1660.

Bogart LJ, O’Donnell P (2018) Multiple long-range inputs evoke NMDA currents in prefrontal cortex fast-spiking interneurons. Neuropsychopharmacology 43:2101-2108.

Caballero A, Tseng KY (2016) GABAergic function as a limiting factor for prefrontal maturation during adolescence. Trends Neurosci 39:441-448.

Caballero A, Flores-Barrera E, Cass DK, Tseng KY (2014a) Differential regulation of parvalbumin and calretinin interneurons in the prefrontal cortex during adolescence. Brain Struct Funct 219:395-406.

Caballero A, Thomases DR, Flores-Barrera E, Cass DK, Tseng KY (2014b) Emergence of GABAergic-dependent regulation of input-specific plasticity in the adult rat prefrontal cortex during adolescence. Psychopharmacology (Berl) 231:1789-1796.

Caballero A, Granberg R, Tseng KY (2016) Mechanisms contributing to prefrontal cortex maturation during adolescence. Neurosci Biobehav Rev 70:4-12.

Caballero A, Flores-Barrera E, Thomases DR, Tseng KY (2020) Downregulation of parvalbumin expression in the prefrontal cortex during adolescence causes enduring prefrontal disinhibition in adulthood. Neuropsychopharmacology. Advance online publication. Retrieved May 13, 2020. doi:10.1038/s41386020-0709-9.

Casey BJ, Giedd JN, Thomas KM (2000) Structural and functional brain development and its relation to cognitive development. Biol Psychol 54:241-257.

Cass DK, Thomases DR, Caballero A, Tseng KY (2013) Developmental disruption of gamma-aminobutyric acid function in the medial prefrontal cortex by noncontingent cocaine exposure during early adolescence. Biol Psychiatry 74:490-501.

Cass DK, Flores-Barrera E, Thomases DR, Vital WF, Caballero A, Tseng KY (2014) CB1 cannabinoid receptor stimulation during adolescence impairs the maturation of GABA function in the adult rat prefrontal cortex. Mol Psychiatry 19:536-543.

Do KQ, Cabungcal JH, Frank A, Steullet P, Cuenod M (2009) Redox dysregulation, neurodevelopment, and schizophrenia. Curr Opin Neurobiol 19:220-230.

Flores-Barrera E, Thomases DR, Heng LJ, Cass DK, Caballero A, Tseng KY (2014) Late adolescent expression of GluN2B transmission in the prefrontal cortex is input-specific and requires postsynaptic protein kinase A and D1 dopamine receptor signaling. Biol Psychiatry 75:508-516.

Flores-Barrera E, Thomases DR, Cass DK, Bhandari A, Schwarcz R, Bruno JP, Tseng KY (2017) Preferential disruption of prefrontal GABAergic function by nanomolar concentrations of the $\alpha 7 \mathrm{nACh}$ negative modulator kynurenic acid. J Neurosci 37:7921-7929.

Gabbott PL, Dickie BG, Vaid RR, Headlam AJ, Bacon SJ (1997) Localcircuit neurones in the medial prefrontal cortex (areas 25, 32 and $24 \mathrm{~b})$ in the rat: morphology and quantitative distribution. J Comp Neurol 377:465-499.

Gilmartin MR, Kwapis JL, Helmstetter FJ (2012) Trace and contextual fear conditioning are impaired following unilateral microinjection of musci$\mathrm{mol}$ in the ventral hippocampus or amygdala, but not the medial prefrontal cortex. Neurobiol Learn Mem 97:452-464

Gilmartin MR, Balderston NL, Helmstetter FJ (2014) Prefrontal cortical regulation of fear learning. Trends Neurosci 37:455-464.

Gogtay N, Vyas NS, Testa R, Wood SJ, Pantelis C (2011) Age of onset of schizophrenia: perspectives from structural neuroimaging studies. Schizophr Bull 37:504-513.

Hardingham GE, Do KQ (2016) Linking early-life NMDAR hypofunction and oxidative stress in schizophrenia pathogenesis. Nat Rev Neurosci 17:125-134

Ishikawa A, Nakamura S (2003) Convergence and interaction of hippocampal and amygdalar projections within the prefrontal cortex in the rat. J Neurosci 23:9987-9995.

Kantrowitz JT, Javitt DC (2010) N-methyl-d-aspartate (NMDA) receptor dysfunction or dysregulation: the final common pathway on the road to schizophrenia? Brain Res Bull 83:108-121.

Kessler RC, Amminger GP, Aguilar-Gaxiola S, Alonso J, Lee S, Ustün TB (2007) Age of onset of mental disorders: a review of recent literature. Curr Opin Psychiatry 20:359-364.

Kinney JW, Davis CN, Tabarean I, Conti B, Bartfai T, Behrens MM (2006) A specific role for NR2A-containing NMDA receptors in the maintenance of parvalbumin and GAD67 immunoreactivity in cultured interneurons. J Neurosci 26:1604-1615.

Milad MR, Wright CI, Orr SP, Pitman RK, Quirk GJ, Rauch SL (2007) Recall of fear extinction in humans activates the ventromedial prefrontal cortex and hippocampus in concert. Biol Psychiatry 62:446-454.

Nakazawa K, Zsiros V, Jiang Z, Nakao K, Kolata S, Zhang S, Belforte JE (2012) GABAergic interneuron origin of schizophrenia pathophysiology. Neuropharmacology 62:1574-1583.

O'Donnell P (2011) Adolescent onset of cortical disinhibition in schizophrenia: insights from animal models. Schizophr Bull 37:484-492.

Paus T, Keshavan M, Giedd JN (2008) Why do many psychiatric disorders emerge during adolescence?. Nat Rev Neurosci 9:947-957.

Phillips RG, LeDoux JE (1992) Differential contribution of amygdala and hippocampus to cued and contextual fear conditioning. Behav Neurosci 106:274-285.

Powell SB, Sejnowski TJ, Behrens MM (2012) Behavioral and neurochemical consequences of cortical oxidative stress on parvalbumin-interneuron maturation in rodent models of schizophrenia. Neuropharmacology 62:1322-1331.

Rotaru DC, Yoshino H, Lewis DA, Ermentrout GB, Gonzalez-Burgos G (2011) Glutamate receptor subtypes mediating synaptic activation of prefrontal cortex neurons: relevance for schizophrenia. J Neurosci 31:142156.

Sierra-Mercado D, Corcoran KA, Lebrón-Milad K, Quirk GJ (2006) Inactivation of the ventromedial prefrontal cortex reduces expression of conditioned fear and impairs subsequent recall of extinction. Eur J Neurosci 24:1751-1758.

Sierra-Mercado D, Padilla-Coreano N, Quirk GJ (2011) Dissociable roles of prelimbic and infralimbic cortices, ventral hippocampus, and basolateral amygdala in the expression and extinction of conditioned fear. Neuropsychopharmacology 36:529-538. 
Sotres-Bayon F, Sierra-Mercado D, Pardilla-Delgado E, Quirk GJ (2012) Gating of fear in prelimbic cortex by hippocampal and amygdala inputs. Neuron 76:804-812.

Steullet P, Cabungcal JH, Monin A, Dwir D, O’Donnell P, Cuenod M, Do KQ (2016) Redox dysregulation, neuroinflammation, and NMDA receptor hypofunction: a "central hub" in schizophrenia pathophysiology? Schizophr Res 176:41-51.

Thomases DR, Cass DK, Tseng KY (2013) Periadolescent exposure to the NMDA receptor antagonist MK-801 impairs the functional maturation of local GABAergic circuits in the adult prefrontal cortex. J Neurosci 33:26-34.

Thomases DR, Cass DK, Meyer JD, Caballero A, Tseng KY (2014) Early adolescent MK-801 exposure impairs the maturation of ventral hippocampal control of basolateral amygdala drive in the adult prefrontal cortex. J Neurosci 34:9059-9066.
Tse MT, Piantadosi PT, Floresco SB (2015) Prefrontal cortical gammaaminobutyric acid transmission and cognitive function: drawing links to schizophrenia from preclinical research. Biol Psychiatry 77:929-939.

Tseng KY, Chambers RA, Lipska BK (2009) The neonatal ventral hippocampal lesion as a heuristic neurodevelopmental model of schizophrenia. Behav Brain Res 204:295-305.

Wang HX, Gao WJ (2010) Development of calcium-permeable AMPA receptors and their correlation with NMDA receptors in fast-spiking interneurons of rat prefrontal cortex. J Physiol 588:28232838 .

Zhang W, Rosenkranz JA (2013) Repeated restraint stress enhances cue-elicited conditioned freezing and impairs acquisition of extinction in an age-dependent manner. Behav Brain Res 248:12-24. 